\title{
Quantization of fields over de Sitter space by the method of generalized coherent states
}

\author{
S A Pol'shin $†$ \\ Department of Physics, Kharkov National University, \\ Svobody Sq., 4, 61077, Kharkov, Ukraine
}

\begin{abstract}
A system of generalized coherent states for the de Sitter group obeying the Klein-Gordon equation and corresponding to the massive spin zero particles over the de Sitter space is considered. This allows us to construct the quantized scalar field by the resolution over these coherent states; the corresponding propagator is computed by the method of analytic continuation to the complex de Sitter space and coincides with expressions obtained previously by other methods. Considering the case of spin $1 / 2$ we establish the connection of the invariant Dirac equation over the de Sitter space with irreducible representations of the de Sitter group. The set of solutions of this equation is obtained in the form of the product of two different systems of generalized coherent states for the de Sitter group. Using these solutions the quantized Dirac field over de Sitter space is constructed and its propagator is found. It is a result of action of some de Sitter invariant spinor operator onto the spin zero propagator with an imaginary shift of a mass. We show that the constructed propagators possess the de Sitter-invariance and causality properties.
\end{abstract}

\section{Introduction}

In the last few years a considerable progress in the theory of massive scalar field over the de Sitter (dS) space is attained due to using the new mathematical methods. In [四] is shown that the two-point Wightman function $\mathcal{W}(x, y)$ which corresponds to this field and obeys the conditions of causality, dS-invariance and positive definiteness can be obtained as a boundary value of the holomorphic function $W\left(z_{1}, z_{2}\right)$ defined over the complex dS space. In turn, the function $W\left(z_{1}, z_{2}\right)$ can be represented as an integral over so-called "plane waves"; these "plane waves" obey the Klein-Gordon equation over the dS space and generalize the usual plane waves over the Minkowski space. In [2] to examine the quantum fields over the $\mathrm{dS}$ space we applied the method of generalized coherent states (CS) which has been fruitfully used in various physical problems (see [3]

† E-mail: it1593@online.kharkov.ua 
and references therein). In the mentioned paper we showed that the above "plane waves" are CS for the dS space to within the coordinate-independent multiplier, and their scalar product coincides with the two-point function considered in [1].

Nevertheless, even in the spin zero case some questions remain open. Can we construct the quantized field by the expansion over the mentioned "plane waves" in such a way that its propagator will be equal to $\mathcal{W}(x, y)-\mathcal{W}(y, x)$ ? What is the explicit form of this propagator? Passing to the case of spin $1 / 2$ we see that usual methods are insufficient at all for the consistent construction of a theory of quantized field over the dS space. Indeed, a lot of papers were concerned with obtaining the solutions of covariant ( [4] and references therein) and group theoretical [5] Dirac equation over the dS space by the method of separation of variables. However, all these solutions have a complicated form which considerably troubles the construction of the theory of quantized field. Only in the little-known paper [6] the summation over one of such a set of solutions was performed; the resulting propagator is not dS-invariant and does not obey the causality principle. On the other hand, in [7] a spinor propagator was found starting from the demands of dS-invariant Dirac equation satisfaction, dS-invariance and the boundary conditions; but the quantized field corresponding to it was not found; on the contrary, in the Anti-de Sitter space the quantized spinor fields with an invariant and causal propagator was constructed long ago [8].

In the present paper we show that all these problems may be solved using the method of generalized coherent states, and build the theory of massive quantized scalar and spinor fields over the $\mathrm{dS}$ space using this method. The present paper is composed as follows. In section 2, bearing in mind the application to the spinor field, we give the method of construction of CS in the maximally general form for which the Perelomov's definition is a special case.

In section 3 we consider the scalar field. In subsection 3.1 we consider the dS space, its symmetry group and the classification of its irreducible representations. The realization of the $\mathrm{dS}$ group as a group of transformations of Lemaitre coordinate system is given too. Following [2], in subsection 3.2 we realize the $\mathrm{dS}$ group as a group of transformations of functions over $\mathbb{R}^{3}$, and then construct the CS system for the dS space which corresponds to the massive spin zero particles and obeys the dS-invariant KleinGordon equation. The scalar product of two CS is the two-point function considered in [1]; from here its dS-invariance follows immediately that is proved in [1] by other reasons. The integral defining this two-point function may be regularized passing to the complex dS space. For the sake of completeness we reproduce some results of the paper by J.Bros and U.Moschella [1] and compute the two-point function over the complex $\mathrm{dS}$ space in the explicit form. In subsection 3.3 we construct the quantized scalar field by the expansion over CS constructed in subsection 3.2. The propagator of this field is the difference of two-point function and the permuted one. We show that the 
boundary value on the real $\mathrm{dS}$ space of the two-point function computed in subsection 3.2 coincides with the Green's function obtained previously by other methods [9, 10]. The propagator which is the difference of two two-point functions coincides with that obtained previously starting from the demands of the dS-invariance and the satisfaction of the Klein-Gordon equation and the boundary conditions [11]. Thus, the relation between different expressions for the propagator available in the literature is established (the review of papers concerning the propagators over the dS space see in [12]).

In section 4 we consider the spinor field. In subsection 4.1 we consider the dSinvariant Dirac equation and show that the corresponding representation of the dS group is irreducible and falls under the classification listed in subsection 3.1. Also we show that this equation admits the reduction to the covariant form by the well simpler way than that proposed previously [7, 13]. In subsection 4.2 we construct the CS system for

the four-spinor representation of the dS group in the form of $4 \times 2$-matrices. Solutions of the dS-invariant Dirac equation are the products of these CS and scalar CS obtained in subsection 3.2. In fact, these solutions are the more compact form of the spinor "plane waves" obtained in [2]. The invariance properties of these solutions allow us to construct of them a dS-invariant two-point function and compute it passing to the complex dS space. In subsection 4.3 we construct the quantized spinor field using these solutions; its propagator is expressed by the boundary values of two-point function obtained in subsection 4.2 and coincides with the expression obtained a priori in [7] to within the constant multiplier.

In section 5 we briefly summarize the results of this paper.

\section{Definition of the CS system}

Let $\mathcal{G}$ is a Lie group and $\mathcal{G} \ni g \mapsto T(g)$ is its representation in a linear vector space $H$ with operators $T(g)$. Consider some vector $\left|\psi_{0}\right\rangle \in H$ yielding the set of vectors

$$
\left\{\left|\psi_{g}\right\rangle \equiv T(g) \psi_{0}, \forall g \in \mathcal{G}\right\}
$$

Define the equivalence relation $\sim$ between the vectors of the $H$ space coordinated with the product over $H$ in the following way. Let $\left|\xi^{\prime}\right\rangle$ and $\left|\xi^{\prime \prime}\right\rangle$ are the vectors of $H$. Then we assume the existence of a product (which, in general, is not the mapping of $H \times H$ to $\mathbb{C}$ ) such as

$$
\left|\xi^{\prime}\right\rangle \sim\left|\xi^{\prime \prime}\right\rangle \Rightarrow\left\langle\xi^{\prime} \mid \xi^{\prime}\right\rangle=\left\langle\xi^{\prime \prime} \mid \xi^{\prime \prime}\right\rangle
$$

Consider the subgroup $\mathcal{H}$ of $\mathcal{G}$ which remains in the rest the equivalency class generated by $\left|\psi_{0}\right\rangle$ :

$$
h \in \mathcal{H} \Longleftrightarrow T(h)\left|\psi_{0}\right\rangle \sim\left|\psi_{0}\right\rangle .
$$

It is obvious that the number of unequivalent elements of the above mentioned set $\left|\psi_{g}\right\rangle$ is less than the number of elements of the group $\mathcal{G}$ because the elements $g$ and 
$g h, h \in \mathcal{H}$ generate the equivalent vectors. Then, in fact, the set of unequivalent vectors is determined by the set of right equivalency classes $g \mathcal{H}$ which compose the symmetric space $\mathcal{G} / \mathcal{H}$.

The mapping $\mathcal{G} / \mathcal{H} \ni \xi \mapsto g_{\xi} \in \mathcal{G}$ such that for an arbitrary $g_{1} \in \mathcal{G}$ the equality

$$
g_{1} g_{\xi}=g_{\xi^{\prime}} h \quad h \in \mathcal{H} \quad \xi^{\prime}=\xi_{g_{1}}
$$

is valid, is called the lifting from the $\mathcal{G} / \mathcal{H}$ space to the $\mathcal{G}$ group, where $\xi \mapsto \xi_{g}$ is the action of $\mathcal{G}$ over the $\mathcal{G} / \mathcal{H}$ space. We shall use the following simple method of construction of liftings. Let $\xi_{\circ}$ is a "standard" point of the $\mathcal{G} / \mathcal{H}$ space. Let us denote as $g_{\xi}$ the set of transformations parametrized by points $\xi$ of the $\mathcal{G} / \mathcal{H}$ space so as $\xi=\left(\xi_{\circ}\right)_{g_{\xi}}$. It is easily seen that $\xi \mapsto g_{\xi}$ is a lifting. Indeed, let $g_{1} \in \mathcal{G}$ is an arbitrary transformation from the group $\mathcal{G}$. Then the transformations $g_{1} g_{\xi}$ and $g_{\xi^{\prime}}$ both transform the point $\xi_{\circ}$ into the point $\xi^{\prime}$; then the transformation $\left(g_{1} g_{\xi}\right)^{-1} g_{\xi^{\prime}}$ remains the point $\xi_{\circ}$ in the rest and therefore belongs to $\mathcal{H}$.

The choice of lifting is the choice of the representative $g_{\xi} \in \mathcal{G}$ for each equivalency class $\xi$. Then the set of all unequivalent vectors $\left|\psi_{g}\right\rangle$ is given by the coherent states system

$$
|\xi\rangle=T\left(g_{\xi}\right)\left|\psi_{0}\right\rangle
$$

The major property of the CS system is its $\mathcal{G}$-invariance which follows from (2.1):

$$
T(g)|\xi\rangle \sim\left|\xi_{g}\right\rangle \quad g \in \mathcal{G}
$$

The Perelomov's definition for the CS system is narrower than ours as he suppose that $\sim$ is the equality to within the phase:

$$
\left|\xi^{\prime}\right\rangle \sim\left|\xi^{\prime \prime}\right\rangle \Leftrightarrow\left|\xi^{\prime}\right\rangle=\mathrm{e}^{\mathrm{i} \alpha}\left|\xi^{\prime \prime}\right\rangle \quad \alpha \in \mathbb{R} .
$$

In a certain sense, our definition is the further generalization of a so-called vectorlike CS [14. Another difference of our definition from Perelomov's one is that we, following [14], do not assume the compactness of the $\mathcal{H}$ subgroup.

\section{Scalar field}

\subsection{Representations of the de Sitter group}

The dS space is a four-dimensional hyperboloid determined by the equation $\eta_{A B} x^{A} x^{B}=$ $-R^{2}$ in the five-dimensional space with the pseudo-euclidean metric $\eta_{A B} \quad(A, B, \ldots=$ $0 \ldots 3,5)$ of signature $(+----)$. Except the explicitly covariant vierbein indices, all the ones are raised and lowered by the Galilean metric tensors $\eta_{A B}$ and $\eta_{\mu \nu}$. The metric in coordinates $x^{\mu}$ has the form

$$
g_{\mu \nu}=\eta_{\mu \nu}-\frac{x^{\mu} x^{\nu}}{R^{2} \chi^{2}} \quad g^{\mu \nu}=\eta^{\mu \nu}+\frac{x^{\mu} x^{\nu}}{R^{2}}
$$


where $\chi=\left(1+x \cdot x / R^{2}\right)^{1 / 2}$. The symmetry group of the $\mathrm{dS}$ space is the $\mathrm{dS}$ group $S O(4,1)$ with ten generators $J^{A B}=-J^{B A}$ obey commutation relations

$$
\left[J_{A B}, J_{C D}\right]=\eta_{A D} J_{B C}+\eta_{B C} J_{A D}-\eta_{A C} J_{B D}-\eta_{B D} J_{A C} .
$$

Let us denote $P^{\mu}=R^{-1} J^{5 \mu}$; these generators correspond to translations.

We denote the action of the arbitrary element $g \in S O(4,1)$ of the dS group over the $\mathrm{dS}$ space as $x \mapsto x_{g}$. The stationary subgroup of an arbitrary point of dS space is $S O(3,1)$; then we can identify the dS space with the set of equivalency classes $S O(4,1) / S O(3,1)$.

Let us construct the operators

$$
\Pi_{i}^{ \pm}=P_{i} \pm \frac{1}{R} J_{0 i}
$$

Using the commutation relations (3.2) it is easy to show that

$$
\left[\Pi_{i}^{+}, \Pi_{k}^{+}\right]=\left[\Pi_{i}^{-}, \Pi_{k}^{-}\right]=0 .
$$

We can take the operators $\boldsymbol{\Pi}^{+}, \boldsymbol{\Pi}^{-}, P^{0}$ and $J_{i k}$ as a new set of generators of the dS group; they generate subgroups which we denote as $\mathcal{T}^{+}, \mathcal{T}^{-}, \mathcal{T}^{0}$ and $\mathcal{R}=S O(3)$, respectively. The groups $\mathcal{T}^{ \pm}$are abelian by the virtue of (3.4). Besides (3.4), the commutation relations are

$$
\begin{aligned}
& {\left[\Pi_{i}^{+}, \Pi_{k}^{-}\right]=-\frac{2}{R} P^{0} \delta_{i k}+\frac{2}{R^{2}} J_{i k} \quad\left[P^{0}, J_{i k}\right]=0} \\
& {\left[\Pi_{i}^{ \pm}, J_{k l}\right]=\Pi_{k}^{ \pm} \delta_{i l}-\Pi_{l}^{ \pm} \delta_{i k} \quad\left[P^{0}, \boldsymbol{\Pi}^{ \pm}\right]= \pm \frac{1}{R} \boldsymbol{\Pi}^{ \pm} .}
\end{aligned}
$$

The dS group has two independent Casimir operators:

$$
C_{2}=-\frac{1}{2 R^{2}} J_{A B} J^{A B} \quad C_{4}=W_{A} W^{A}
$$

where

$$
W_{A}=\frac{1}{8 R} \varepsilon_{A B C D E} J^{B C} J^{D E}
$$

is an analog of the Pauly-Lubanski pseudovector in the Poincaré group. There are two series of the dS group irreducible representations [15]:

1) $\boldsymbol{\pi}_{p, q}, p=1 / 2,1,3 / 2, \ldots ; q=p, p-1, \ldots, 1$ and $1 / 2$. The eigenvalues of Casimir operators in this series are

$$
\begin{aligned}
& R^{2} C_{2}=p(p+1)+q(q-1)-2 \\
& R^{2} C_{4}=p(p+1) q(q-1) .
\end{aligned}
$$

2) $\boldsymbol{\nu}_{m, s}$. The quantity $s$ is a spin, $s=0,1 / 2,1, \ldots$; the quantity $m$ corresponds to a mass, at the integer spin $m^{2}>0$; at the half-integer spin $m^{2}>1 / 4 R^{2}$; at $s=0 \quad m^{2}>-2 / R^{2}$.

$$
\begin{aligned}
& C_{2}=-m^{2}+R^{-2}(s(s+1)-2) \\
& C_{4}=-m^{2} s(s+1) .
\end{aligned}
$$


The generators of five-dimensional rotations are

$$
J^{(l) A B}=\left(x^{A} \eta^{B C}-x^{B} \eta^{A C}\right) \partial_{C} .
$$

As the fifth coordinate is not independent: $x^{5}=R \chi$, then $\partial_{5}=0$ and we obtain the generators of scalar representation:

$$
P_{\mu}^{(l)}=\chi \partial_{\mu} \quad J^{(l) \mu \nu}=\left(x^{\mu} \eta^{\nu \sigma}-x^{\nu} \eta^{\mu \sigma}\right) \partial_{\sigma} .
$$

They compose the representation $\boldsymbol{\nu}_{m, 0}$ since

$$
W_{A}^{(l)}=0 \Rightarrow C_{4}^{(l)}=0 .
$$

As $(-g)^{1 / 2}=1 / \chi$, then for the second order Casimir operator in the scalar representation we obtain from (3.6) and (3.11):

$$
C_{2}^{(l)}=\square \equiv(-g)^{-1 / 2} \partial_{\mu}\left((-g)^{1 / 2} g^{\mu \nu} \partial_{\nu}\right) .
$$

Then using (3.9) we obtain that in the representation $\boldsymbol{\nu}_{m, 0}$ the Klein-Gordon equation

$$
\left(\square+m^{2}+2 R^{-2}\right) \psi=0
$$

is satisfied.

By the virtue of (3.4) in the scalar representation the generators (3.3) are the derivatives along certain new coordinates called the Lemaitre coordinates:

$$
\boldsymbol{\Pi}^{ \pm}=\frac{\partial}{\partial \boldsymbol{y}_{ \pm}}
$$

Substituting (3.11) and (3.14) into r.h.s. and l.h.s of equation (3.3) respectively, we obtain the connection of $\boldsymbol{y}_{ \pm}$with $x^{\mu}$. We denote a new time coordinate independent on $\boldsymbol{y}_{ \pm}$as $y_{ \pm}^{0}=\tau_{ \pm}$; then the transformation rules from the old coordinates to the new ones are

$$
\boldsymbol{y}_{ \pm}=\boldsymbol{x} \mathrm{e}^{\mp \tau_{ \pm} / R} \quad \mathrm{e}^{ \pm \tau_{ \pm} / R}=\chi \pm \frac{x^{0}}{R}
$$

The operator $P_{0}$ in the new coordinates takes the form

$$
P_{0}^{(l)}=\frac{\partial}{\partial \tau_{ \pm}} \mp \frac{1}{R} \boldsymbol{y}_{ \pm} \frac{\partial}{\partial \boldsymbol{y}_{ \pm}}
$$

The finite transformations belonging to the subgroups $\mathcal{T}^{ \pm}$and $\mathcal{T}^{0}$, which we denote as $\Theta_{ \pm}$and $\Theta_{0}$ respectively, act in the scalar representation by the following way:

$$
\begin{aligned}
& g=\Theta_{ \pm}(\boldsymbol{a}) \equiv \exp \left(\boldsymbol{\Pi}^{ \pm} \boldsymbol{a} R\right):\left\{\begin{array}{l}
\boldsymbol{y}_{ \pm} \longmapsto \boldsymbol{y}_{ \pm}^{\prime}=\boldsymbol{y}_{ \pm}+\boldsymbol{a} R \\
\tau_{ \pm} \longmapsto \tau^{\prime} \tau_{ \pm}
\end{array}\right. \\
& g=\Theta_{0}(\varepsilon) \equiv \exp \left(P_{0} \varepsilon R\right):\left\{\begin{array}{l}
\boldsymbol{y}_{ \pm}^{\prime}=\boldsymbol{y}_{ \pm} \mathrm{e}^{\mp \varepsilon} \\
\tau_{ \pm}^{\prime}=\tau_{ \pm}+\varepsilon R .
\end{array}\right.
\end{aligned}
$$

We assume that the transformations act in the order from the right to the left. 


\subsection{Scalar coherent states}

The dS group is isomorphic to the group of conformal transformations of the threedimensional real space. We denote the vector of this space as $\boldsymbol{w}$. There exist two different conformal realizations of the dS group; the first one corresponds to the upper sign, and the second one to the lower one in the following formulas. The generators take the form

$$
\begin{array}{rlrl}
R \boldsymbol{\Pi}^{\mp} & =-\frac{\partial}{\partial \boldsymbol{w}} & R \boldsymbol{\Pi}^{ \pm} & =w^{2} \frac{\partial}{\partial \boldsymbol{w}}-2 \boldsymbol{w}\left(\boldsymbol{w} \frac{\partial}{\partial \boldsymbol{w}}\right) \\
R P_{0}= \pm \boldsymbol{w} \frac{\partial}{\partial \boldsymbol{w}} & J_{i k}=w_{k} \frac{\partial}{\partial w_{i}}-w_{i} \frac{\partial}{\partial w_{k}} .
\end{array}
$$

They obey commutation relations (3.4) and (3.5). Finite transformations have the form

$$
\begin{aligned}
& g=\Theta_{\mp}(\boldsymbol{a}): \boldsymbol{w}_{g}=\boldsymbol{w}-\boldsymbol{a} \\
& g=\Theta_{ \pm}(\boldsymbol{a}): \boldsymbol{w}_{g}=\frac{\boldsymbol{w}+\boldsymbol{a} w^{2}}{1+2 \boldsymbol{w} \boldsymbol{a}+w^{2} a^{2}} \\
& g=\Theta_{0}(\varepsilon): \boldsymbol{w}_{g}=\boldsymbol{w} \mathrm{e}^{ \pm \varepsilon} .
\end{aligned}
$$

Let us define two different representations of the $\mathrm{dS}$ group acting over the space of functions dependent on $\boldsymbol{w}$ :

$$
T_{\sigma}^{ \pm}(g) f(\boldsymbol{w})=\left(\alpha_{\boldsymbol{w}}^{ \pm}(g)\right)^{\sigma} f\left(\boldsymbol{w}_{g^{-1}}\right)
$$

where $\sigma \in \mathbb{C}$ and

$$
\alpha_{\boldsymbol{w}}^{ \pm}(g)=\operatorname{det}\left(\frac{\partial w_{g^{-1}}^{i}}{\partial w^{k}}\right)^{-1 / 3}= \begin{cases}1 & g \in \mathcal{T}^{\mp} \mathrm{S} \mathcal{R} \\ \mathrm{e}^{ \pm \varepsilon} & g=\Theta_{0}(\varepsilon) \\ 1-2 \boldsymbol{a} \boldsymbol{w}+a^{2} w^{2} & g=\Theta_{ \pm}(\boldsymbol{a}) .\end{cases}
$$

We denote these representations as $T_{\sigma}^{ \pm}$. It is easily seen that the generators in these representations are

$$
\begin{aligned}
& R \boldsymbol{\Pi}^{\mp}=-\frac{\partial}{\partial \boldsymbol{w}} \quad R \boldsymbol{\Pi}^{ \pm}=w^{2} \frac{\partial}{\partial \boldsymbol{w}}-2 \boldsymbol{w}\left(\boldsymbol{w} \frac{\partial}{\partial \boldsymbol{w}}\right)+2 \sigma \boldsymbol{w} \\
& R P_{0}= \pm\left(\boldsymbol{w} \frac{\partial}{\partial \boldsymbol{w}}-\sigma\right) \quad J_{i k}=w_{k} \frac{\partial}{\partial w_{i}}-w_{i} \frac{\partial}{\partial w_{k}} .
\end{aligned}
$$

We define the scalar product in the space of representation $T_{\sigma}^{ \pm}$as follows:

$$
\left\langle f_{1} \mid f_{2}\right\rangle=\int_{\mathbb{R}^{3}} \mathrm{~d}^{3} \boldsymbol{w} f_{1}^{*}(\boldsymbol{w}) f_{2}(\boldsymbol{w}) .
$$

It is not difficult to show that it is dS-invariant at

$$
\sigma=\sigma_{0} \equiv-\frac{3}{2}-\mathrm{i} \mu R \quad \mu \in \mathbb{R}
$$

Then the representation $T_{\sigma_{0}}^{ \pm}$is unitary; but it is reducible since we do not assume the square integrability of functions carrying it and therefore the space contains the invariant 
subspace of square integrable functions. Such an extension of the representation space is necessary for the construction of CS with noncompact stability subgroups [14].

The equality

$$
g_{y_{ \pm}}=\Theta_{ \pm}\left(\boldsymbol{y}_{ \pm} / R\right) \Theta_{0}\left(\tau_{ \pm} / R\right)
$$

defines the lifting in the sense of (2.1) since the transformation $g_{y_{ \pm}}$transforms the origin into the point with coordinates $y_{ \pm}$. As an equivalency relation we can take the equality. Then the vector $\left|\psi_{0}\right\rangle$ being a Lorentz-invariant under the action of the representation $T_{\sigma}^{ \pm}$is $\left|\psi_{0}\right\rangle=\left(1-w^{2}\right)^{\sigma}$. Then we can construct the CS system

$$
|x, \pm ; \sigma\rangle=T_{\sigma}^{ \pm}\left(g_{y_{ \pm}(x)}\right)\left|\psi_{0}\right\rangle
$$

where we assume that the Lemaitre coordinates are dependent on $x^{\mu}$ by the transformations (3.15). The explicit form of CS as a function of $\boldsymbol{w}$ is

$$
|x, \pm ; \sigma\rangle \equiv \Phi_{\boldsymbol{w}}^{(0) \pm}(x ; \sigma)=\left(1-w^{2}\right)^{\sigma} \varphi_{k \boldsymbol{w}}^{(0) \pm}(x ; \sigma)
$$

where

$$
\varphi_{k}^{(0) \pm}(x ; \sigma)=\left(\chi \pm \frac{k \cdot x}{R}\right)^{\sigma} \quad k_{\boldsymbol{w}}^{\mu}=\left(\frac{1+w^{2}}{1-w^{2}}, \pm \frac{2 \boldsymbol{w}}{1-w^{2}}\right)
$$

then $k_{\boldsymbol{w}} \cdot k_{\boldsymbol{w}}=1$. From (2.2) the transformation rules

$$
\Phi_{\boldsymbol{w}}^{(0) \pm}\left(x_{g} ; \sigma\right)=\left(\alpha_{\boldsymbol{w}}^{ \pm}(g)\right)^{\sigma} \Phi_{\boldsymbol{w}^{\prime}}^{(0) \pm}(x ; \sigma) \quad \boldsymbol{w}^{\prime}=\boldsymbol{w}_{g^{-1}}
$$

follow.

The functions $\varphi_{k}^{(0) \pm}\left(x ; \sigma_{0}\right)$ obey the dS-invariant Klein-Gordon equation (3.13) and were known previously in this capacity [1, [1]. Under $R \rightarrow \infty$ these functions pass into the usual plane waves over the Minkowski space.

Let us consider the scalar product of two CS in the representation $T_{\sigma_{0}}^{ \pm}$; it is easily seen that the scalar products in the representations $T_{\sigma_{0}}^{+}$and $T_{\sigma_{0}}^{-}$are equal to each other. This may be proved considering the inversion $\boldsymbol{w} \mapsto-\boldsymbol{w} / w^{2}$ which yields

$$
\Phi_{\boldsymbol{w}}^{(0) \pm}\left(x ; \sigma_{0}\right) \mapsto\left(-w^{2}\right)^{-\sigma_{0}} \Phi_{\boldsymbol{w}}^{(0) \mp}\left(x ; \sigma_{0}\right) .
$$

Then a two-point function can be defined as

$$
\left\langle\stackrel{2}{x}, \pm ; \sigma_{0} \mid \stackrel{1}{x}, \pm ; \sigma_{0}\right\rangle=\int_{\mathbb{R}^{3}} \mathrm{~d}^{3} \boldsymbol{w} \Phi_{\boldsymbol{w}}^{(0) \pm}\left(\stackrel{1}{x} ; \sigma_{0}\right) \Phi_{\boldsymbol{w}}^{(0) \pm}\left(\stackrel{2}{x} ; \sigma_{0}^{*}\right)=\frac{1}{8} \mathcal{W}^{(0)}(\stackrel{1}{x}, \stackrel{2}{x})
$$

It is dS-invariant by the virtue of unitarity of the representation $T_{\sigma_{0}}^{ \pm}$:

$$
\mathcal{W}^{(0)}\left(\stackrel{1}{x}_{g}, \stackrel{2}{x}_{g}\right)=\mathcal{W}^{(0)}(\stackrel{1}{x}, \stackrel{2}{x}) \quad g \in S O(4,1) .
$$

We can obtain an another expression for $\mathcal{W}^{(0)}(\stackrel{1}{x}, \stackrel{2}{x})$ passing to the integration over 3 -sphere [1]. Let us consider the unit euclidean four-vector $l_{a}, a, b=1,2,3,5$ dependent on the three-vector $\boldsymbol{w}$ :

$$
l_{\boldsymbol{w}}^{a}=\left(\mp \frac{2 \boldsymbol{w}}{1+w^{2}}, \frac{1-w^{2}}{1+w^{2}}\right) \quad l_{\boldsymbol{w}}^{a} l_{\boldsymbol{w}}^{a}=1 .
$$


Then computing the Jacobian of the transformation from $\boldsymbol{w}$ to $\boldsymbol{l}_{\boldsymbol{w}}$ we obtain

$$
\mathcal{W}^{(0)}(\stackrel{1}{x}, \stackrel{2}{x})=\int_{S^{3}} \frac{\mathrm{d}^{3} \boldsymbol{l}}{l^{5}}\left(\frac{x^{0}+l^{a} \stackrel{1}{x}}{R}\right)^{-\mathrm{i} \mu R-3 / 2}\left(\frac{\stackrel{x}{x}^{0}+l^{a} \stackrel{2}{x^{a}}}{R}\right)^{\mathrm{i} \mu R-3 / 2} .
$$

The function $\mathcal{W}^{(0)}(\stackrel{1}{x}, \stackrel{2}{x})$ coincides with the two-point function over the dS space considered in [1]. In general, the integral (3.20) diverges because $\left|\psi_{0}\right\rangle$ is not squareintegrable over $\mathbb{R}^{3}$. We can make the integral meaningful passing to the complex dS space with subsequent computation of the boundary values over the real dS space [1]. Then the dS-invariance of two-point function remains valid since the transformation rules (3.19) remains correct.

Let us consider the domain $\mathcal{D}^{ \pm}$in the complex dS space (we shall denote its points as $\zeta$ ) defined as

$$
\pm \operatorname{Im} \zeta^{0}>0 \quad \operatorname{Im} \zeta^{A} \operatorname{Im} \zeta_{A}>0 .
$$

The domain $\mathcal{D}^{+}\left(\mathcal{D}^{-}\right)$is the domain of analyticity of the functions $\varphi_{k}^{(0) \pm}\left(\zeta ; \sigma_{0}\right)$ $\left(\varphi_{k}^{(0) \pm}\left(\zeta ; \sigma_{0}^{*}\right)\right)$. Then the integral (3.20) converges at $\stackrel{1}{\zeta} \in \mathcal{D}^{+}$and $\stackrel{2}{\zeta} \in \mathcal{D}^{-}$since the 3 -sphere volume is finite. Let us choose the points as

$$
\stackrel{1}{\zeta}^{A}=(\mathrm{i} \cosh v, \mathbf{0}, i \sinh v) \quad \stackrel{2}{\zeta}^{A}=(-\mathrm{i}, \mathbf{0}, 0) \quad v \in \mathbb{R} .
$$

Then using the formula [17]

$$
{ }_{2} F_{1}(a, b ; c ; z)=\frac{2^{1-c} \Gamma(c)}{\Gamma(b) \Gamma(c-b)} \int_{0}^{\pi} \mathrm{d} \varphi \frac{(\sin \varphi)^{2 b-1}(1+\cos \varphi)^{c-2 b}}{\left(1-\frac{z}{2}+\frac{z}{2} \cos \varphi\right)^{a}}
$$

we obtain

$$
\left.\mathcal{W}^{(0)} \stackrel{1}{\zeta}, \stackrel{2}{\zeta}\right)=\frac{\pi^{2}}{2} \mathrm{e}^{-\pi \mu R}{ }_{2} F_{1}\left(-\sigma_{0}^{*},-\sigma_{0} ; 2 ; \frac{1-\rho}{2}\right)
$$

where $\rho=R^{-2} \stackrel{1}{\zeta}^{A} \stackrel{2}{\zeta}_{A}$. The expression obtained in [1] is in fact equivalent to the above expression to within a constant multiplier.

\subsection{Quantized spin zero field}

Let us define the quantized spin zero field in the dS space as

$$
\phi^{(0)}(x)=\int_{\mathbb{R}^{3}} \mathrm{~d}^{3} \boldsymbol{w}\left(\Phi_{\boldsymbol{w}}^{(0)+}\left(x ; \sigma_{0}\right) a^{(+)}(\boldsymbol{w})+\Phi_{\boldsymbol{w}}^{(0)-}\left(x ; \sigma_{0}^{*}\right) a^{(-) \dagger}(\boldsymbol{w})\right)
$$

where $a^{( \pm)}(\boldsymbol{w})$ and $a^{( \pm) \dagger}(\boldsymbol{w})$ are two sets of bosonic creation-annihilation operators with the commutation relations

$$
\left[a^{( \pm)}(\boldsymbol{w}), a^{( \pm) \dagger}\left(\boldsymbol{w}^{\prime}\right)\right]=\delta\left(\boldsymbol{w}, \boldsymbol{w}^{\prime}\right)
$$


where $\delta\left(\boldsymbol{w}_{1}, \boldsymbol{w}_{2}\right)$ is the $\delta$-function over $\mathbb{R}^{3}$ and all other commutators vanish. Now compute the propagator

$$
\left[\phi^{(0)}(\stackrel{1}{x}), \phi^{(0) \dagger}(\stackrel{2}{x})\right] \equiv \frac{1}{8} G^{(0)}(\stackrel{1}{x}, \stackrel{2}{x})=\frac{1}{8}\left(\mathcal{W}^{(0)}(\stackrel{1}{x}, \stackrel{2}{x})-\mathcal{W}^{(0)}(\stackrel{2}{x}, \stackrel{1}{x})\right)
$$

in the explicit form by passing to the complex dS space. Considering the real dS space as a boundary of the domains $(\stackrel{1}{\zeta}, \stackrel{2}{\zeta}) \in \mathcal{D}^{ \pm} \times \mathcal{D}^{\mp}$, let us denote the boundary values of the function $\mathcal{W}^{(0)}(\stackrel{1}{\zeta}, \stackrel{2}{\zeta})$ as $\mathcal{W}^{(0) \pm}(\stackrel{1}{x}, \stackrel{2}{x})$. To compute these boundary values we put $\stackrel{1}{\zeta}=\stackrel{1}{x}+\mathrm{i} \stackrel{1}{\epsilon}$ and $\stackrel{2}{\zeta}=\stackrel{2}{x}-\mathrm{i} \stackrel{2}{\epsilon}$, where $\stackrel{1}{\epsilon}$ and $\stackrel{2}{\epsilon}$ are two real infinitesimal time-like forward four-vectors and then indeed $(\stackrel{1}{\zeta}, \stackrel{2}{\zeta}) \in \mathcal{D}^{+} \times \mathcal{D}^{-}$. It is easily seen that

$$
\stackrel{1}{\zeta}^{A} \stackrel{2}{\zeta}_{A}=x_{x}^{A} \stackrel{2}{x}_{A}+\frac{\mathrm{i}}{x^{5}{ }_{x^{5}}^{2}}(\stackrel{1}{\epsilon}+\stackrel{2}{\epsilon}) \cdot\left(\frac{2}{x} \underset{x^{5}}{-}-\frac{\stackrel{x}{x}}{x^{5}}\right) .
$$

Then under the above assumptions the sign of the imaginary part of $\stackrel{1}{\zeta}^{A} \stackrel{2}{\zeta}_{A}$ does not depend on the way in which $\stackrel{1}{\epsilon}$ and $\stackrel{2}{\epsilon}$ tend to zero. Let $\stackrel{2}{x}^{\mu}=0$ and $\stackrel{1}{x} \cdot \stackrel{1}{x} \geq 0$, then

$$
\stackrel{1}{\zeta}^{A} \stackrel{2}{\zeta}_{A}=\stackrel{1}{x}^{A} \stackrel{2}{x}_{A}-\mathrm{i} 0 \varepsilon\left(\stackrel{1}{x}^{0}\right)
$$

The case of backward $\stackrel{1}{\epsilon}$ and $\stackrel{2}{\epsilon}$ (then $\left.(\stackrel{1}{\zeta}, \stackrel{2}{\zeta}) \in \mathcal{D}^{-} \times \mathcal{D}^{+}\right)$may be considered in the completely analogous way. Then

$$
\mathcal{W}^{(0) \pm}(\stackrel{1}{x}, \stackrel{2}{x})=\frac{\pi^{2}}{2} \mathrm{e}^{-\pi \mu R}{ }_{2} F_{1}\left(-\sigma_{0}^{*},-\sigma_{0} ; 2 ; \frac{1-G \pm \mathrm{i} 0 \varepsilon\left({ }^{0} x^{0}\right)}{2}\right)
$$

where $G=R^{-2} \stackrel{1}{x}^{A} \stackrel{2}{x}_{A}$. As $(\stackrel{1}{\zeta}, \stackrel{2}{\zeta}) \in \mathcal{D}^{+} \times \mathcal{D}^{-}$yields $(\stackrel{2}{\zeta}, \stackrel{1}{\zeta}, \zeta) \in \mathcal{D}^{-} \times \mathcal{D}^{+}$, then we get

$$
\mathcal{W}^{(0)+}(\stackrel{1}{x}, \stackrel{2}{x})=\mathcal{W}^{(0)-}(\stackrel{2}{x}, \stackrel{1}{x})
$$

(cf. proposition 2.2 of [1]) and by the virtue of (3.23) the propagator is equal to

$$
G^{(0)}(\stackrel{1}{x}, \stackrel{2}{x})=\mathcal{W}^{(0)+}(\stackrel{1}{x}, \stackrel{2}{x})-\mathcal{W}^{(0)-}(\stackrel{1}{x}, \stackrel{2}{x}) .
$$

The function $\mathcal{W}^{(0)-}(\stackrel{1}{x}, \stackrel{2}{x})$ coincides to within the constant multiplier with the propagator obtained in [9] starting from the demands of satisfaction of the Klein-Gordon equation and the boundary conditions. This function may be obtained by the summation over the modes [10] and by the method of discrete lattice [18] too.

As we assume that $\stackrel{1}{x} \cdot \stackrel{1}{x} \geq 0$ then $\frac{1-G}{2} \geq 1$, but the integral (3.22) with which we define the hypergeometric function, makes no sense at $z \in[1,+\infty)$ and then demands the analytic continuation in the domain which contains the mentioned interval. To this 
end we shall use the formulas [17

$$
\begin{aligned}
& u_{1}=\frac{\Gamma(c) \Gamma(b-a)}{\Gamma(c-a) \Gamma(b)} u_{3}+\frac{\Gamma(c) \Gamma(a-b)}{\Gamma(c-b) \Gamma(a)} u_{4} \\
& u_{2}=\frac{\Gamma(a+b+1-c) \Gamma(b-a)}{\Gamma(b+1-c) \Gamma(b)} \mathrm{e}^{\mp \mathrm{i} \pi a} u_{3}+\frac{\Gamma(a+b+1-c) \Gamma(a-b)}{\Gamma(a+1-c) \Gamma(a)} \mathrm{e}^{\mp \mathrm{i} \pi b} u_{4}
\end{aligned}
$$

where the upper or lower sign should be chosen depending on the $\operatorname{Im} z$ greater or smaller than zero, and $u_{1}, \ldots, u_{4}$ are the Kummer solutions of the hypergeometric equation:

$$
\begin{aligned}
& u_{1}={ }_{2} F_{1}(a, b ; c ; z) \\
& u_{2}={ }_{2} F_{1}(a, b ; a+b+1-c ; 1-z) \\
& u_{3}=(-z)^{-a}{ }_{2} F_{1}\left(a, a+1-c ; a+1-b ; z^{-1}\right) \\
& u_{4}=(-z)^{-b}{ }_{2} F_{1}\left(b, b+1-c ; b+1-a ; z^{-1}\right) .
\end{aligned}
$$

The functions $u_{1}, u_{3}, u_{4}$ are holomorphic at $z<0$. Then at $a+b+1=2 c$

$$
\left.u_{2}\right|_{z-\mathrm{i} 0} ^{z+\mathrm{i} 0}=\mathrm{i}\left(\mathrm{e}^{\pi \mu R}+\mathrm{e}^{-\pi \mu R}\right) \theta(-z) u_{1} \quad z \neq 0 .
$$

To obtain the behavior of $u_{2}$ at $z=0$ we shall use the formula [17]

$$
\begin{aligned}
{ }_{2} F_{1}(a, b ; a+b & -m ; z) \frac{1}{\Gamma(a+b-m)} \\
= & \frac{\Gamma(m)(1-z)^{-m}}{\Gamma(a) \Gamma(b)} \sum_{n=0}^{m-1} \frac{(a-m)_{n}(b-m)_{n}}{(1-m)_{n} n !}(1-z)^{n} \\
& +\frac{(-1)^{m}}{\Gamma(a-m) \Gamma(b-m)} \sum_{n=0}^{\infty} \frac{(a)_{n}(b)_{n}}{(n+m)_{n} n !}\left[\bar{h}_{n}-\ln (1-z)\right](1-z)^{n}
\end{aligned}
$$

which holds at $|\arg (1-z)|<\pi$, and the formulas

$$
\begin{aligned}
& \left.z^{-1}\right|_{z-\mathrm{i} 0} ^{z+\mathrm{i} 0}=-2 \pi \mathrm{i} \delta(z) \\
& \Gamma\left(\frac{1}{2}+z\right) \Gamma\left(\frac{1}{2}-z\right)=\frac{\pi}{\cos (\pi z)} .
\end{aligned}
$$

Then using (3.26) we obtain

$$
\begin{aligned}
{ }_{2} F_{1}\left(-\sigma_{0},-\sigma_{0}^{*} ; 2 ; z\right) & \left.\right|_{z-\mathrm{i} 0} ^{z+\mathrm{i} 0}=-\mathrm{i}\left(\mathrm{e}^{\pi \mu R}+\mathrm{e}^{-\pi \mu R}\right) \\
& \times\left(\frac{\delta(1-z)}{\mu^{2}+\left(4 R^{2}\right)^{-1}}-\theta(z-1){ }_{2} F_{1}\left(-\sigma_{0},-\sigma_{0}^{*} ; 2 ; 1-z\right)\right) .
\end{aligned}
$$

Putting together the above expression and (3.24) we finally obtain

$$
\begin{aligned}
G^{(0)}(\stackrel{1}{x}, \stackrel{2}{x})=-\mathrm{i} \pi^{2}\left(1+\mathrm{e}^{-2 \pi \mu R}\right) \varepsilon\left(\stackrel{1}{x}^{0}-\stackrel{2}{x}^{0}\right) \\
\times\left[\frac{\delta(1+G)}{\mu^{2}+\left(4 R^{2}\right)^{-1}}-\frac{1}{2} \theta\left(-\frac{1+G}{2}\right){ }_{2} F_{1}\left(-\sigma_{0},-\sigma_{0}^{*} ; 2 ; \frac{1+G}{2}\right)\right] .
\end{aligned}
$$

To within the constant multiplier, the above expression coincides with that obtained previously starting from the demands of satisfaction of the Klein-Gordon equation and the boundary conditions [11]. 


\section{Spinor field}

\subsection{The Dirac equation}

Introducing the matrices

$$
\gamma^{5}=\mathrm{i} \gamma^{0} \gamma^{1} \gamma^{2} \gamma^{3} \quad \tilde{\gamma}^{\mu}=-\mathrm{i} \gamma^{5} \gamma^{\mu} \quad \tilde{\gamma}^{5}=\mathrm{i} \gamma^{5}
$$

we can write down the generators of the four-spinor representation of the dS group in the five-dimensional form:

$$
J^{(s) A B}=\frac{1}{4}\left[\tilde{\gamma}^{A}, \tilde{\gamma}^{B}\right] .
$$

The equalities

$$
\begin{aligned}
& \tilde{\gamma}^{A} \tilde{\gamma}^{B}+\tilde{\gamma}^{B} \tilde{\gamma}^{A}=2 \eta^{A B} \\
& \tilde{\gamma}^{A} \tilde{\gamma}^{B} \tilde{\gamma}^{C}=\eta^{A B} \tilde{\gamma}^{C}+\eta^{B C} \tilde{\gamma}^{A}-\eta^{A C} \tilde{\gamma}^{B}+\frac{1}{2} \varepsilon^{A B C D E} \tilde{\gamma}_{D} \tilde{\gamma}_{E} \\
& \tilde{\gamma}^{A} \tilde{\gamma}^{B} \tilde{\gamma}^{C} \tilde{\gamma}^{D}=\eta^{A B} \tilde{\gamma}^{C} \tilde{\gamma}^{D}+\eta^{B C} \tilde{\gamma}^{A} \tilde{\gamma}^{D}-\eta^{A C} \tilde{\gamma}^{B} \tilde{\gamma}^{D}+ \\
& +2\left(\eta^{A D} J^{(s) B C}+\eta^{C D} J^{(s) A B}-\eta^{B D} J^{(s) A C}\right)-\varepsilon^{A B C D E} \tilde{\gamma}_{E}
\end{aligned}
$$

hold. With the help of the above expressions and (3.6), (3.7) we obtain

$$
R^{2} C_{2}^{(s)}=5 / 2 \quad W_{A}^{(s)}=\frac{3}{4} \tilde{\gamma}_{A} \quad R^{2} C_{4}^{(s)}=\frac{45}{16} .
$$

Comparing the above expression with (3.8) we see that it is the representation $\boldsymbol{\pi}_{3 / 2,3 / 2}$. We shall choose the standard form of $\gamma$-matrices. Then it is easy to show that the explicit form of generators is

$$
\begin{array}{ll}
\boldsymbol{\Pi}^{+(s)}=\frac{1}{R}\left(\begin{array}{rr}
0 & -\boldsymbol{\sigma} \\
0 & 0
\end{array}\right) & \boldsymbol{\Pi}^{-(s)}=\frac{1}{R}\left(\begin{array}{cc}
0 & 0 \\
\boldsymbol{\sigma} & 0
\end{array}\right) \\
P^{0(s)}=\frac{1}{2}\left(\begin{array}{rr}
1 & 0 \\
0 & -1
\end{array}\right) & J_{i k}^{(s)}=-\mathrm{i} \varepsilon_{i k l}\left(\begin{array}{ll}
\sigma^{l} & 0 \\
0 & \sigma^{l}
\end{array}\right) .
\end{array}
$$

We denote matrices of finite transformations as $U(g)$. Then we obtain

$$
\begin{aligned}
& U\left(\Theta_{ \pm}(\boldsymbol{a})\right)=1-\boldsymbol{\Pi}^{ \pm(s)} \boldsymbol{a} R \\
& U\left(\Theta_{0}(\varepsilon)\right)=\exp \left(-P_{0}^{(s)} \varepsilon\right) .
\end{aligned}
$$

Let us consider the representation $\boldsymbol{\pi}_{3 / 2,3 / 2} \otimes \boldsymbol{\nu}_{m, 0}$. Its generators are the sum of generators (3.11) (orbital part) and generators (4.1) (spin part). Then the second-order Casimir operator is equal to

$$
C_{2}=C_{2}^{(l)}+C_{2}^{(s)}-R^{-2} J^{(s) A B} J_{A B}^{(l)}
$$


Denoting $\hat{\nabla}_{\mathrm{dS}}=-R^{-1} J^{(s) A B} J_{A B}^{(l)}$ we obtain

$$
C_{2}=\square+\frac{\hat{\nabla}_{\mathrm{dS}}}{R}+\frac{5}{2 R^{2}} .
$$

To compute the fourth-order Casimir operator we write according to (3.12) and (4.5):

$$
R W_{A}=-\frac{1}{8} \varepsilon_{A B C D E} \tilde{\gamma}^{B} \tilde{\gamma}^{C} J^{(l) D E}+\frac{3}{4} \tilde{\gamma}_{A}
$$

For squaring $W_{A}$ it is necessary to use formulas (4.2)-(4.4). The result obtained

$$
C_{4}=\frac{3}{4} \square+\frac{3 \hat{\nabla}_{\mathrm{dS}}}{4 R}+\frac{45}{16 R^{2}}
$$

is in agreement with (3.10) at $s=1 / 2$ and (4.7). From the second Shur's lemma follows that the operators $\hat{\nabla}_{\mathrm{dS}}$ and $\square$ should have fixed eigenvalues in the irreducible representations. Then using (3.9), (4.7) and the equality

$$
\hat{\nabla}_{\mathrm{dS}}^{2}=\frac{1}{4 R^{2}} \tilde{\gamma}^{A} \tilde{\gamma}^{B} \tilde{\gamma}^{C} \tilde{\gamma}^{D} J_{A B}^{(l)} J_{C D}^{(l)}=\square-3 \hat{\nabla}_{\mathrm{dS}} / R
$$

we obtain the quadratic equation for eigenvalues of $\hat{\nabla}_{\mathrm{dS}}$. Solving it yields

$$
\begin{aligned}
& \hat{\nabla}_{\mathrm{dS}}=-2 R^{-1} \pm \mathrm{i} \mu \\
& \square=-\mu^{2} \mp \mathrm{i} R^{-1} \mu-2 R^{-2}
\end{aligned}
$$

where $\mu^{2}=m^{2}-\frac{1}{4 R^{2}}$. As $m^{2}>1 / 4 R^{2}$ (see subsection 3.1), then $\mu$ is a real number. The appearance of two signs indicates that two identical irreducible representations have appeared:

$$
\boldsymbol{\nu}_{m, 0} \otimes \boldsymbol{\pi}_{3 / 2,3 / 2}=2 \boldsymbol{\nu}_{m, 1 / 2}
$$

Using (3.11) we can write

$$
\hat{\nabla}_{\mathrm{dS}}=\Gamma^{\mu} \partial_{\mu} \quad \Gamma^{\mu}=\chi \gamma^{\mu}+\frac{1}{2 R}\left[\gamma^{\mu}, \gamma_{\nu}\right] x^{\nu} .
$$

Choosing the representation which corresponds to the lower sign in (4.8) we finally obtain the group theoretical Dirac equation over the dS space:

$$
\mathrm{i} \Gamma^{\mu} \partial_{\mu} \psi-\left(\mu-2 \mathrm{i} R^{-1}\right) \psi=0 .
$$

This was well known previously out of the context of dS group irreducible representations [19].

The above equation admits the transformation into the covariant form. To this end let us perform the transformation $\Psi=V \psi$, where

$$
V=\left(1-\varepsilon_{\mu} \varepsilon^{\mu}\right)^{-1 / 2}\left(1+\gamma_{\mu} \varepsilon^{\mu}\right) \quad \varepsilon^{\mu}=\frac{x^{\mu}}{R(\chi+1)} .
$$


Then (4.9) passes into

$$
\mathrm{i} V \Gamma^{\mu} V^{-1}\left(\partial_{\mu} \Psi+\left(V \partial_{\mu} V^{-1}\right) \Psi\right)-\left(\mu-2 \mathrm{i} R^{-1}\right) \Psi=0 .
$$

It is easy to show that

$$
\begin{aligned}
& V \Gamma^{\mu} V^{-1}=e_{(\nu)}^{\mu} \gamma^{\nu} \\
& \partial_{\mu}+V \partial_{\mu} V^{-1}=\mathcal{D}_{\mu}-\frac{1}{2 R} \gamma_{\mu}
\end{aligned}
$$

where $e_{\nu}^{(\mu)}$ is the vierbein which is orthonormal with respect to the metric (3.1):

$$
e^{(\mu) \nu}=\eta^{\mu \nu}+\frac{x^{\mu} x^{\nu}}{R^{2}(\chi+1)}
$$

and $\mathcal{D}_{\mu}$ is the spinor covariant derivative

$\mathcal{D}_{(\mu)}=e_{(\mu)}^{\nu} \partial_{\nu}-\frac{1}{2} J^{(s) \nu \rho} G_{\nu \rho \mu} \quad G_{\nu \rho \mu}=e_{(\nu) ; \kappa}^{\sigma} e_{(\rho) \sigma} e_{(\mu)}^{\kappa}=\frac{1}{R^{2}(\chi+1)}\left(x_{\nu} \eta_{\mu \rho}-x_{\rho} \eta_{\nu \mu}\right)$.

Then putting together (4.10)-(4.11) we finally obtain

$$
\mathrm{i} \gamma^{\mu} e_{(\mu)}^{\nu} \mathcal{D}_{\nu} \Psi=\mu \Psi
$$

Another more complicated ways of transformation of (Anti-)dS-invariant Dirac equation to the covariant one were proposed in [7, 13].

\subsection{Spinor coherent states}

In [2] was shown that the solutions of equation (4.9) may be obtained in the form of the product of scalar CS with an imaginary shift of a mass, and basic Dirac 4-spinors. However, there is not the natural action of the full dS group over these 4-spinors. This difficulty may be overcome if we consider $4 \times 2$-matrices whose columns are the mentioned 4-spinors. Indeed, let us denote the constant $4 \times 2$-matrices as $A, A^{\prime}, A^{\prime \prime}$ and define over such matrices the weak equivalence relation $\sim$ and the strong one $\simeq$ as

$$
\begin{array}{ll}
A^{\prime} \sim A^{\prime \prime} \Leftrightarrow A^{\prime}=A^{\prime \prime} B & B \in G L(2, \mathbb{C}) \\
A^{\prime} \simeq A^{\prime \prime} \Leftrightarrow A^{\prime}=A^{\prime \prime} B & B \in S U(2) .
\end{array}
$$

Also define the product of two $4 \times 2$-matrices $A^{\prime}$ and $A^{\prime \prime}$ as $A^{\prime} \overline{A^{\prime \prime}}$, where the upper line denotes the Dirac conjugation. Consider the left action of four-spinor representation of the dS group over these matrices: $g: A \mapsto U(g) A$. It is easy to show that the matrices

$$
|+\rangle=\left(\begin{array}{c}
I_{2} \\
0_{2}
\end{array}\right) \quad|-\rangle=\left(\begin{array}{c}
0_{2} \\
I_{2}
\end{array}\right)
$$

(where $I_{2}$ is the unit $2 \times 2$-matrix) are invariant under transformations which belong to the subgroups $\mathcal{K}^{ \pm} \equiv \mathcal{T}^{ \pm} \mathrm{S}\left(\mathcal{T}^{0} \otimes \mathcal{R}\right)$ to within the weak equivalence relation. In the terms of the strong equivalence relation we have

$$
\left.U(h)| \pm\rangle \simeq\left(\alpha_{\boldsymbol{v}}^{ \pm}(h)\right)^{-1 / 2}\right|_{\boldsymbol{v}=\mathbf{o}}| \pm\rangle \quad h \in \mathcal{K}^{ \pm}
$$


From the other hand, it is easily seen that the subgroups $\mathcal{K}^{ \pm}$are the stability subgroups of the vector $\boldsymbol{w}=\mathbf{o}$ concerning the conformal action (3.17) of the dS group. This allows us to identify the $S O(4,1) / \mathcal{K}^{ \pm}$space with the space $\mathbb{R}^{3}$ of vectors $\boldsymbol{w}$. As the lifting from the $S O(4,1) / \mathcal{K}^{ \pm}$space to the dS group we shall take the transformation which transforms the origin into the point $\boldsymbol{w}$ :

$$
S O(4,1) / \mathcal{K}^{ \pm} \ni \boldsymbol{w} \mapsto g_{\boldsymbol{w}}=\Theta_{\mp}(-\boldsymbol{w}) \in S O(4,1) .
$$

Then the CS system for the $S O(4,1) / \mathcal{K}^{ \pm}$space being dS-invariant to within the weak equivalence relation is

$$
\begin{array}{ll}
|\boldsymbol{w} \pm\rangle & =U\left(g_{\boldsymbol{w}}\right)| \pm\rangle \\
|\boldsymbol{w}+\rangle & =\left(\begin{array}{l}
I_{2} \\
\boldsymbol{\sigma} \boldsymbol{w}
\end{array}\right) \quad|\boldsymbol{w}-\rangle=\left(\begin{array}{l}
-\boldsymbol{\sigma} \boldsymbol{w} \\
I_{2}
\end{array}\right) .
\end{array}
$$

With the help of (2.1) the transformation properties of these vectors with respect to the strong equivalence relation may be written as

$$
\left.U\left(g_{1}\right)|\boldsymbol{w} \pm\rangle \simeq\left(\alpha_{\boldsymbol{v}}^{ \pm}\left(g_{\boldsymbol{w}^{\prime}}^{-1} g_{1} g_{\boldsymbol{w}}\right)\right)^{-1 / 2}\right|_{\boldsymbol{v}=\mathbf{o}}\left|\boldsymbol{w}_{g_{1}} \pm\right\rangle \quad g_{1} \in \mathcal{G} .
$$

As the transformations $T_{\sigma}^{ \pm}(g)$ compose a representation of the dS group then

$$
\alpha_{\boldsymbol{v}}^{ \pm}\left(g_{2} g_{1}\right)=\alpha_{\boldsymbol{v}}^{ \pm}\left(g_{2}\right) \alpha_{\boldsymbol{v}^{\prime}}^{ \pm}\left(g_{1}\right) \quad g_{1}, g_{2} \in \mathcal{G} \quad \boldsymbol{v}^{\prime}=\boldsymbol{v}_{g_{2}^{-1}}
$$

Then using the above expression and (2.1) we get

$$
\alpha_{\boldsymbol{v}}^{ \pm}\left(g_{1}\right)=\alpha_{\boldsymbol{v}^{\prime}}^{ \pm}\left(g_{\boldsymbol{w}^{\prime}}^{-1} g_{1} g_{\boldsymbol{w}}\right) \quad \boldsymbol{v}^{\prime}=\boldsymbol{v}_{g_{\boldsymbol{w}^{\prime}}^{-1}} \quad \boldsymbol{w}^{\prime}=\boldsymbol{w}_{g_{1}}
$$

Putting $\boldsymbol{v}=\boldsymbol{w}^{\prime}$ in the above expression, we can rewrite the transformation properties (4.12) as

$$
\left(\alpha_{\boldsymbol{w}}^{ \pm}(g)\right)^{1 / 2} U(g)\left|\boldsymbol{w}_{g^{-1}} \pm\right\rangle \simeq|\boldsymbol{w} \pm\rangle \quad g \in \mathcal{G} .
$$

It is easy to show that the equalities

$$
\begin{aligned}
& \left(\gamma \cdot k_{\boldsymbol{w}} \mp 1\right)|\boldsymbol{w} \pm\rangle=0 \\
& |\boldsymbol{w} \pm\rangle\langle\boldsymbol{w} \pm|=\frac{1-w^{2}}{2}\left(\gamma \cdot k_{\boldsymbol{w}} \pm 1\right)
\end{aligned}
$$

are correct. Now let us construct the $4 \times 2$-matrix functions

$$
\Phi_{\boldsymbol{w}}^{(1 / 2) \pm}(x)=\Phi_{\boldsymbol{w}}^{(0) \pm}\left(x ; \sigma_{0}-1 / 2\right)|\boldsymbol{w} \pm\rangle .
$$

Using (4.14) we obtain that they obey (4.9):

$$
\left(\mathrm{i} \hat{\nabla}_{\mathrm{dS}}-\mu+2 \mathrm{i} R^{-1}\right) \Phi_{\boldsymbol{w}}^{(1 / 2) \pm}(x)=0 .
$$

These solutions are much simpler that those obtained by the method of separation of variables [4, 5]. 
From the transformation properties (3.19) and (4.13) it follows that under the transformations from the $\mathrm{dS}$ group the functions $\Phi_{\boldsymbol{w}}^{(1 / 2) \pm}(x)$ transform just as the functions $\Phi_{\boldsymbol{w}}^{(0) \pm}\left(x ; \sigma_{0}\right)$, to within the constant matrix transformation:

$$
\Phi_{\boldsymbol{w}}^{(1 / 2) \pm}\left(x_{g}\right) \simeq\left(\alpha_{\boldsymbol{w}}^{ \pm}(g)\right)^{\sigma_{0}} U(g) \Phi_{\boldsymbol{w}^{\prime}}^{(1 / 2) \pm}(x)
$$

where $\boldsymbol{w}^{\prime}=\boldsymbol{w}_{g^{-1}}$. As the inversion $\boldsymbol{w} \mapsto-\boldsymbol{w} / w^{2}$ yields

$$
\Phi_{-\boldsymbol{w} / w^{2}}^{(1 / 2) \pm}(x) \simeq-i\left(-w^{2}\right)^{-\sigma_{0}} \Phi_{\boldsymbol{w}}^{(1 / 2) \mp}(x)
$$

then the functions $\Phi_{\boldsymbol{w}}^{(1 / 2)+}(x)$ and $\Phi_{\boldsymbol{w}}^{(1 / 2)-}(x)$ yield the same two-point function. Let us define it as follows:

$\frac{1}{8} \mathcal{W}^{(1 / 2)}(\stackrel{1}{x}, \stackrel{2}{x})=\int_{\mathbb{R}^{3}} \mathrm{~d}^{3} \boldsymbol{w} \Phi_{\boldsymbol{w}}^{(1 / 2)+}(\stackrel{1}{x}) \bar{\Phi}_{\boldsymbol{w}}^{(1 / 2)+}(\stackrel{2}{x})=\int_{\mathbb{R}^{3}} \mathrm{~d}^{3} \boldsymbol{w} \Phi_{\boldsymbol{w}}^{(1 / 2)-}(\stackrel{1}{x}) \bar{\Phi}_{\boldsymbol{w}}^{(1 / 2)-}(\stackrel{2}{x})$.

From (4.16) follows that it is dS-invariant in the sense that at $g \in \mathcal{G}$

$$
\mathcal{W}^{(1 / 2)}\left(\stackrel{1}{x}_{g}, \stackrel{2}{x}_{g}\right)=U(g) \mathcal{W}^{(1 / 2)}(\stackrel{1}{x}, \stackrel{2}{x}) \bar{U}(g)
$$

Using (4.15) it is easy to show that

$\mathcal{W}^{(1 / 2)}(\stackrel{1}{x}, \stackrel{2}{x})=\frac{1}{2} \int_{S^{3}} \frac{\mathrm{d}^{3} \boldsymbol{l}}{l^{5}}\left(\frac{\stackrel{1}{x}^{0}+l^{a} \stackrel{1}{x^{a}}}{R}\right)^{-\mathrm{i} \mu R-2}\left(\frac{\stackrel{2}{x}^{0}+l^{a} \stackrel{2}{x}^{a}}{R}\right)^{\mathrm{i} \mu R-2}\left(\gamma^{0}+\gamma \boldsymbol{l}+l^{5}\right)$.

As the functions $\Phi_{\boldsymbol{w}}^{(1 / 2) \pm}(\zeta)$ inherit the analyticity properties of functions $\varphi_{k}^{(0) \pm}\left(\zeta ; \sigma_{0}\right)$ over the complex dS space, then, similarly to in the spin zero case, the function $\mathcal{W}^{(1 / 2)}(\stackrel{1}{x}, \stackrel{2}{x})$ converges at $\left(\begin{array}{c}1 \\ \zeta\end{array} \stackrel{2}{\zeta}\right) \in \mathcal{D}^{+} \times \mathcal{D}^{-}$. Choosing the points according to (3.21) and using the equality

$$
\begin{aligned}
& \int_{0}^{\pi} \mathrm{d} \theta \sin ^{2} \theta \cos \theta(\cosh v+\sinh v \cos \theta)^{-\mathrm{i} \mu R-2}=\frac{\pi}{\sinh v} \\
& \times\left({ }_{2} F_{1}\left(1-\frac{\mathrm{i} \mu R}{2}, \frac{1+\mathrm{i} \mu R}{2} ; 2 ;-\sinh ^{2} v\right)-\cosh v_{2} F_{1}\left(1+\frac{\mathrm{i} \mu R}{2}, \frac{1-\mathrm{i} \mu R}{2} ; 2 ;-\sinh ^{2} v\right)\right)
\end{aligned}
$$

we obtain

$$
\begin{aligned}
\mathcal{W}^{(1 / 2)}(\stackrel{1}{\zeta}, \stackrel{2}{\zeta})= & \frac{\pi^{2} \mathrm{e}^{-\pi \mu R}}{\mu-\mathrm{i} R^{-1}} \\
& \times \tilde{\gamma}_{A} \stackrel{1}{\zeta}^{A}\left(\mathrm{i} \hat{\nabla}_{\mathrm{dS}}-\mu+\mathrm{i} R^{-1}\right){ }_{2} F_{1}\left(2-\mathrm{i} \mu R, 1+\mathrm{i} \mu R ; 2 ; \frac{1-\rho}{2}\right) \gamma^{5}
\end{aligned}
$$

where the operator $\hat{\nabla}_{\mathrm{dS}}$ acts onto the coordinates $\stackrel{1}{\zeta}$.

\subsection{Quantized spinor field}

To construct a quantized spinor field, let us use the equality

$$
R^{-1} \tilde{\gamma}^{A} x_{A}\left(\mathrm{i} \hat{\nabla}_{\mathrm{dS}}-\mu+2 \mathrm{i} R^{-1}\right) R^{-1} \tilde{\gamma}^{B} x_{B}=\mathrm{i} \hat{\nabla}_{\mathrm{dS}}+\mu+2 \mathrm{i} R^{-1}
$$


From here follows that if the function $\psi$ obey the Dirac equation (4.9), then the function $\tilde{\gamma}^{A} x_{A} \psi$ obey the same equation with the opposite sign of $\mu$. Then the functions

$$
\tilde{\Phi}_{\boldsymbol{w}}^{(1 / 2) \pm}(x)=R^{-1} \tilde{\gamma}^{A} x_{A} \Phi_{\boldsymbol{w}}^{(0) \pm}\left(x ; \sigma_{0}^{*}-1 / 2\right)|\boldsymbol{w} \pm\rangle
$$

obey equation (4.9). Let us introduce also two sets of fermionic creation-annihilation operators $b^{( \pm)}(\boldsymbol{w})$ and $b^{( \pm) \dagger}(\boldsymbol{w})$, which at the same time are the matrices of dimensionality $2 \times 1$ and $1 \times 2$, respectively, and obey the anticommutation relations

$$
\left\{b^{( \pm)}(\boldsymbol{w}), b^{( \pm) \dagger}\left(\boldsymbol{w}^{\prime}\right)\right\}=\delta\left(\boldsymbol{w}, \boldsymbol{w}^{\prime}\right)\left(\begin{array}{cc}
1 & 0 \\
0 & 1
\end{array}\right)
$$

and all other anticommutators vanish. Then we can construct the quantized spinor field as

$$
\phi^{(1 / 2)}(x)=\int_{\mathbb{R}^{3}} \mathrm{~d}^{3} \boldsymbol{w}\left(\Phi_{\boldsymbol{w}}^{(1 / 2)+}(x) b^{(+)}(\boldsymbol{w})+\tilde{\Phi}_{\boldsymbol{w}}^{(1 / 2)-}(x) b^{(-)}(\boldsymbol{w})\right) .
$$

Using (4.17) it is easy to show that the two-point function which corresponds to the solutions $\widetilde{\Phi}_{\boldsymbol{w}}^{(1 / 2) \pm}(x)$ is equal to

$$
\begin{aligned}
& \left.\int_{\mathbb{R}^{3}} \mathrm{~d}^{3} \boldsymbol{w} \tilde{\Phi}_{\boldsymbol{w}}^{(1 / 2) \pm}(\stackrel{1}{\zeta}) \overline{\tilde{\Phi}}_{\boldsymbol{w}}^{(1 / 2) \pm} \stackrel{2}{\zeta}\right) \\
= & \left.R^{-2} \tilde{\gamma}^{A} \stackrel{1}{\zeta}_{A} \mathcal{W}^{(1 / 2)}(\stackrel{2}{\zeta}, \stackrel{1}{\zeta})\left(\gamma^{5} \tilde{\gamma}^{B} \stackrel{2}{\zeta}_{B} \gamma^{5}\right)=-\mathcal{W}^{(1 / 2)} \stackrel{1}{(} \stackrel{2}{\zeta}, \stackrel{\zeta}{\zeta}\right) .
\end{aligned}
$$

Further, the hypergeometric functions in the r.h.s. of (4.17) differ from $\mathcal{W}^{(0)}(\stackrel{1}{\zeta}, \stackrel{2}{\zeta})$ only by the constant multiplier and the imaginary shift of mass: $\mu \rightarrow \mu+i R^{-1}$. Then computing the difference of its values on the edges of the cut $z \in[1,+\infty)$ we can use (3.27). Passing to the boundary values equation (4.20) yields

$$
R^{-2} \tilde{\gamma}^{A} \stackrel{1}{x_{A}} \mathcal{W}^{(1 / 2)+}(\stackrel{2}{x}, \stackrel{1}{x})\left(\gamma^{5} \tilde{\gamma}^{B} \stackrel{2}{x_{B}} \gamma^{5}\right)=-\mathcal{W}^{(1 / 2)-}(\stackrel{1}{x}, \stackrel{2}{x})
$$

which is analogous to Equation (3.25) for the spin zero case. Then using (3.27) for the spin $1 / 2$ propagator

$$
\left\{\phi_{\alpha}^{(1 / 2)}(\stackrel{1}{x}), \bar{\phi}_{\beta}^{(1 / 2)}(\stackrel{2}{x})\right\} \equiv \frac{1}{8} G_{\alpha \beta}^{(1 / 2)}(\stackrel{1}{x}, \stackrel{2}{x})=\frac{1}{8}\left(\mathcal{W}_{\alpha \beta}^{(1 / 2)+}(\stackrel{1}{x}, \stackrel{2}{x})-\mathcal{W}_{\alpha \beta}^{(1 / 2)-}(\stackrel{1}{x}, \stackrel{2}{x})\right)
$$

where $\alpha, \beta=1, \ldots, 4$ are spinor indices, we finally obtain

$$
\begin{aligned}
G^{(1 / 2)}(\stackrel{1}{x}, \stackrel{2}{x})= & \frac{2 \pi^{2}}{\mu-\mathrm{i} R^{-1}}\left(1-\mathrm{e}^{-2 \pi \mu R}\right) \varepsilon\left({ }^{1} x^{0}-\stackrel{2}{x}^{0}\right) \tilde{\gamma}_{A}{ }^{1} x^{A}\left(\mathrm{i} \hat{\nabla}_{\mathrm{dS}}-\mu+\mathrm{i} R^{-1}\right) \\
& \times\left[\frac{\delta(1+G)}{\mu\left(\mu+\mathrm{i} R^{-1}\right)}-\frac{1}{2} \theta\left(-\frac{1+G}{2}\right){ }_{2} F_{1}\left(2-\mathrm{i} \mu R, 1+\mathrm{i} \mu R ; 2 ; \frac{1+G}{2}\right)\right] \gamma^{5},
\end{aligned}
$$

where the operator $\hat{\nabla}_{\mathrm{dS}}$ acts onto the coordinates $\stackrel{1}{x}$. The above expression coincides with the solution of Cauchy problem for the Dirac equation over the dS space obtained in [7], to within a constant multiplier. 


\section{Concluding remarks}

To summarize the results of this paper, we can say that the CS method allow us to quantize massive spin 0 and $1 / 2$ fields over the $\mathrm{dS}$ space in the uniform way. Both in the spin zero case and in the spin $1 / 2$ one the starting-point is the invariant wave equations which correspond to irreducible representations of the dS group. The solutions of these equations are constructed using CS for the dS group; in the spin zero case the dS-invariant Klein-Gordon equation is satisfied by the scalar CS itself. In the spin 1/2 case the solutions of dS-invariant Dirac equation are constructed from two different CS systems which correspond to different representations of the dS group and different stationary subgroups. Both in the spin zero case and in the spin $1 / 2$ one these sets of solutions possess the same transformation properties under the dS group, with only difference that the constant matrix transformation is added in the spin $1 / 2$ case.

From these sets of solutions we can construct the two-point functions $\mathcal{W}^{(s)}(\stackrel{1}{x}, \stackrel{2}{x})$ which have the following properties:

(i) dS-invariance:

$$
\mathcal{W}^{(1 / 2)}\left(\stackrel{1}{x}_{g}, \stackrel{2}{x}_{g}\right)=U_{s}(g) \mathcal{W}^{(1 / 2)}(\stackrel{1}{x}, \stackrel{2}{x}) \bar{U}_{s}(g)
$$

where $U_{s}(g)$ is the identical representation at $s=0$ and the four-spinor representation at $s=1 / 2$.

(ii) Causality:

$$
\mathcal{W}^{(s)}(\stackrel{1}{x}, \stackrel{2}{x})=\mathcal{W}^{(s)}(\stackrel{2}{x}, \stackrel{1}{x}) \quad \stackrel{1}{x}_{A} \stackrel{2}{x}^{A}>-R^{2}
$$

(iii) Regularized function $\mathcal{W}^{(s)}(\stackrel{1}{x}, \stackrel{2}{x})$ is the boundary value of the function $\mathcal{W}^{(s)}(\stackrel{1}{\zeta}, \stackrel{2}{\zeta})$ which is analytic in certain domain of the complex dS space.

For the spin zero case the above properties were proved in [1]; but also in this case the CS method gives the sufficient simplification since the property 1 is found almost obvious. Defining the creation-annihilation operators so that they possess the necessary (anti)commutation relations, we can construct the quantized fields $\phi^{(s)}(x)$ using the mentioned sets of solutions; the propagators of these fields are equal to

$$
\left[\phi^{(s)}(\stackrel{1}{x}), \bar{\phi}^{(s)}(\stackrel{2}{x})\right]_{ \pm}=\frac{1}{8}\left(\mathcal{W}^{(s)}(\stackrel{1}{x}, \stackrel{2}{x})-\mathcal{W}^{(s)}(\stackrel{2}{x}, \stackrel{1}{x})\right)
$$

and therefore are dS-invariant and causal automatically.

\section{Acknowledgments}

I am grateful to Yu P Stepanovsky for the constant support during the work and to W Drechsler and $\mathrm{Ph}$ Spindel sending me copies of their papers [16, 12, 6]. 


\section{References}

[1] Bros J and Moschella U 1996 Rev. Math. Phys. 8327

[2] Pol'shin S A 1999 Phys. Lett. B 44956

[3] Perelomov A M 1986 Generalized Coherent States and Their Applications (Berlin: Springer); Twareque Ali S, Antoine J-P, Gazeau J-P and Mueller U A 1995 Rev. Math. Phys. 71013

[4] Villalba V M 1993 Modern Physics Letters A 8 2351; Cotăescu I 1998 Modern Physics Letters A 132991

[5] Chernikov N A and Shavokhina N S 1973 Teor. Mat. Fiz. 1677

[6] Schomblond C and Spindel Ph 1976 Bulletin de l'Académie royale de Belgique (Classe des Sciences), 5eme Série LXII 124

[7] Shavokhina N S 1972 Teor. Mat. Fiz. 10412

[8] Fronsdal C and Haugen R B 1975 Phys.Rev.D 123810

[9] Candelas P and Raine D J 1975 Phys. Rev. D 12965

[10] Birrell N D and Davies P C W 1982 Quantum fields in curved space (Cambridge: Cambridge University Press)

[11] Chernikov N A and Tagirov E A 1968 Ann. Inst. Henry Poincaré, Sect. A IX 109; Börner G and Durr H P 1969 Nuovo Cimento A 64 669; Gutzwiller M 1956 Helv. Phys. Acta 29313

[12] Gabriel Cl and Spindel Ph 1997 J.Math.Phys. 38 622; Allen B and Jacobson Th 1986 Commun. Math. Phys. 103669

[13] Gath E 1989 Class. Quant. Grav. 6745

[14] Papaloucas L C, Rembieliński J and Tybor W 1989 J.Math.Phys. 302407

[15] Dixmier J 1961 Bulletin de la Societe Mathematique de France 89 9; Kihlberg A and Ström S 1965 Arkiv för fysik $\mathbf{3 1} 491$

[16] Drechsler W and Prugovečki E 1991 Found. Phys. 21 513; Trucks M 1996 Class. Quant. Grav. 13 2941

[17] Erdélyi A, Magnus W, Oberhettinger F and Tricomi F 1953 Higher transcendental functions (New York: Mc Graw-Hill) Vol. 1

[18] Huang J J and Wang M J 1988 Nuovo Cimento A 100723

[19] Gürsey F 1963 Relativity, Groups and Topology eds. de Witt C. and de Witt B. (New York: Gordon and Breach) p 89 